\title{
Mutations of rpob Gene Associated with Rifampin Resistance among Mycobacterium Tuberculosis Isolated in Tuberculosis Regional Reference Laboratory in Northeast of Iran during 2015-2016
}

\author{
Ashraf Tavanaee sani ${ }^{1}$, Habib Ashna ${ }^{2}$, Afsaneh Kaffash ${ }^{2}$, Azad Khaledi ${ }^{3,4}$, \\ Kiarash Ghazvini ${ }^{5 *}$
}

OPEN ACCESS

Citation: Ashraf Tavanaee sani, Habib Ashna, Afsaneh Kaffash, Azad Khaledi ${ }^{3,4}$, Kiarash Ghazvini. Mutations of rpob Gene Associated with Rifampin Resistance among Mycobacterium Tuberculosis Isolated in Tuberculosis Regional Reference Laboratory in Northeast of Iran during 2015-2016. Ethiop J Health Sci.2017;28(3):299. doi:http://dx.doi.org/10.4314/ejhs.v27i5.7 Received: November 30, 2017

Accepted: December 3, 2017

Published: May 1, 2018

Copyright: (C) 2018 Tavanaee, A. et al. This is an open access article distributed under the terms of the Creative Commons Attribution License, which permits unrestricted use, distribution, and reproduction in any medium, provided the original author and source are credited. Funding: Nil Nil

Competing Interests: The authors declare that this manuscript was approved by all authors in its form and that no competing interest exists.

Affiliation and Correspondence:

${ }^{1}$ Infectious Diseases Department, Mashhad University of Medical Sciences, Mashhad, Iran

${ }^{2}$ Infectious Diseases Department, Mashhad University of Medical Sciences, Mashhad, Iran

${ }^{3}$ Infectious Diseases Research Center, Kashan University of Medical Sciences, Kashan, IR Iran ${ }^{4}$ Department of Microbiology and Immunology, School of Medicine, Kashan University of Medical Sciences, Kashan, Iran

${ }^{5}$ Antimicrobial Resistance Research Center, Mashhad University of

Medical Sciences, Mashhad, Iran

*Email: Ghazvinik@mums.ac.ir

\section{ABSTRACT}

BACKGROUND: Drug resistance is a leading concern in control of TB. Resistance against rifampin as one of the most important drugs in the treatment of Mycobacterium tuberculosis is caused by mutations in the 81-base pair region of the rpoB gene encoding the $\beta$-subunit of RNA polymerase. This study aimed to characterize the mutations in the rpoB gene associated with rifampin resistance among M. tuberculosis.

METHODS: This study was conducted on referred samples of patients who did not respond to anti-TB treatment, in Tuberculosis Regional Reference Laboratory at Shariati Hospital. Drug susceptibility of M. tuberculosis isolates was surveyed using a proportional method on $\mathrm{LJ}$ medium. The isolates with resistant to rifampin were reconfirmed and then the rpoB gene was amplified and sequenced.

RESULTS: Among 27 resistant cases, 8, 11 and 8 people were from Iran, Afghanistan, and Turkmenistan, respectively. In 26 out of 27 isolates, rpoB gene mutations were observed. The most prevalent mutations belonged to the codon 53. The most prevalent mutations belonged to the TCG (Ser) 531TTG (leu) with prevalence 51.8\% $(n=14)$, and GAC (Asp)516TAC (Tyr), CAC (His) 526GAC (Asp) and CAC (His) 526TAC(Tyr) mutations with prevalence $14.8 \%(\mathrm{n}=4)$. Twenty-three isolates had just one mutation.

CONCLUSION: The use of rpoB gene sequencing led to the lack of the need for growth of the organism in the culture medium, the direct use of clinical samples, reduction of biological risks and a detection about $96.3 \%$ of $M D R T B$ cases lowering the cost of the treatment.

KEYWORDS: Drug-resistant, Mycobacterium tuberculosis, rpoB gene, Rifampicin 


\section{INTRODUCTION}

TB infects one-third of the world's population, and nearly two million people die annually due to tuberculosis(1). Drug resistance is emerging globally, half a million new cases of MDR-TB (Multi-Drug-Resistance TB) were seen each year, and drug resistance is the main concern in control of TB. Since 1994, WHO reported the resistance to tuberculosis regularly. MDR and XDR-TB cause high mortality (more than 90\%) in patients with HIV (2-4).

Therefore, information collected of susceptibility to anti-TB drugs and surveillance and analysis of drug-resistant to $\mathrm{TB}$ in any region is useful in tracing of MDR and XDR-TB strains. Treatment of MDR is complicated and requires using toxic drugs with fewer side effects, and long duration of treatment can cause a serious reduction in the likelihood of successful treatment.

Prompt treatment with anti-TB drugs and supportive therapy with corticosteroids reduces the rate of death and disability caused by $\mathrm{TB}$, but this disease is still life-threatening and it is a major cause of death in some parts of the world (5). In 2015 , there were an estimated 10.4 million new TB cases over the world, of which 5.9 million (56\%), 3.5 million (34\%) and 1.0 million (10\%) were among men, women, and children, respectively. As well, it was reported 480000 new cases of multidrug-resistant TB (MDR-TB) and an additional 100000 people with rifampicinresistant TB (RR-TB) who were also newly eligible for MDR-TB treatment(6). Rifampin resistance in $M$. tuberculosis usually occurs by a mutation in $r p o B$ gene encoding the beta subunit of RNA polymerase. Although a single point mutation is sufficient to provide resistance to rifampin, many cases of $r p o B$ multiple mutants in M. tuberculosis have been reported (6). Drug susceptibility testing (DST) using culture techniques of $M$. tuberculosis needs at least 7-10 days, and sometimes takes 8-12 weeks for a final decision, and this time frame is inappropriate for management of patients and leads to an increase in MDR-TB strains. Hence, rapid, inexpensive and simple techniques for tracing the drug-resistant tuberculosis are required to screening patients at the high risk for MDR-TB. Of these techniques can be mentioned to the rapid molecular methods based on amplification of DNA (NAA) in order to follow the mutations in genes responsible for resistance to rifampin $(r p o B)$, isoniazid (inhA, kat $G$ ) and ofloxacin (gyrA) (7). In most studies conducted on rpoB gene, to investigate the mutations of resistance to rifampicin drug, many different mutations were seen in codon $81 \mathrm{bp}$ in diverse regions of the gene, but in a number of cases, have been reported without any mutation in this region. Such cases show that in addition to mutation, other mechanisms are involved in resistance to this drug. At the same time, it supports this concept that furthermore to the $r p o B$ gene, one or more other genes also have contributed in resistance to the rifampin. It is possible that the occurrence of multiple mutations in different genes has a combined effect on resistance. Although various studies have been conducted in this field worldwide, no such study has ever been carried out in the northeast of Iran, and it is important to have information from all parts of the world. Therefore, this study aimed to characterize the mutations in the rpoB gene associated with rifampin resistance among $M$. tuberculosis isolated in Tuberculosis Regional Reference Laboratory in the northeast of Iran during 2015-2016.

\section{METHODS}

This cross-sectional study was conducted in 20152016 on samples of patients referred to Tuberculosis Regional Reference Laboratory at Shariati Hospital in the Northeast of Iran. These patients did not respond to anti-TB treatment. $M$. tuberculosis strains were isolated and identified using Ziehl Neelsen staining; growth rate, colony morphology, pigment production, nitrate reduction, catalase, niacin tests and amplification of IS6110. H37RV strain of M. tuberculosis was considered as control. Drug susceptibility testing for isolates was performed using a proportional method on the Lowenstein Jensen (LJ) medium. For reconfirmation of rifampin susceptibility pattern, samples were cultured on LJ medium containing a certain concentration of rifampin

DOI: http://dx.doi.org/10.4314/ejhs.v28i3.7 
antibiotic (susceptibility was determined at a concentration of $40 \mathrm{mg} / \mathrm{ml}$ for rifampin), and after incubation for 4-6 weeks in $37^{\circ} \mathrm{C}$, the results were evaluated. In this method, when the colony growth in the medium containing antibiotic was equal or more than $1 \%$ of colonies numbers in the medium without antibiotic, it was considered as a rifampin-resistant strain. Isolates of $M$. tuberculosis which were resistant to rifampin were stored for further investigations. Patient demographic details such as age, gender, the onset of disease symptoms, duration of disease, duration of drug consumption and the treatment process were recorded by a physician for estimating any relationship with resistance. After confirming by phenotyping tests, genomic DNA was extracted by a CTAB protocol as described previously by van Soolingen and et al. (8). Briefly, $1.5 \mathrm{ml}$ of the concentrated culture was heated at $80^{\circ} \mathrm{C}$ for 20 min to kill the cells and centrifuged. Then the cells were resuspended in $500 \mathrm{ul}$ of TE buffer $(0.01 \mathrm{M}$ Tris-HCl, $0.001 \mathrm{M}$ EDTA [pH 8.0]). Lysozyme was added to a final concentration of $1 \mathrm{mg} / \mathrm{ml}$, and the tube was incubated for $1 \mathrm{~h}$ at $37^{\circ} \mathrm{C}$. Seventy microliters of $10 \%$ sodium dodecyl sulfate (SDS) and $6 \mathrm{ul}$ of proteinase $\mathrm{K}$ (at a $10-\mathrm{mg} / \mathrm{ml}$ concentration) were added, and the mixture was incubated for $10 \mathrm{~min}$ at $65^{\circ} \mathrm{C}$. An 80 -pul volume of N-cetyl-N, N, N-trimethyl ammonium bromide was added. The cups were vortexed briefly and incubated for $10 \mathrm{~min}$ at $65^{\circ} \mathrm{C}$. An equal volume of chloroform-isoamyl alcohol $(24: 1, \mathrm{vol} / \mathrm{vol})$ was added, and the mixture was vortexed for $10 \mathrm{~s}$. After centrifugation for $5 \mathrm{~min}, 0.6$ volume of isopropanol was added to the supernatant to precipitate the DNA. After $30 \mathrm{~min}$ at $-20^{\circ} \mathrm{C}$ and centrifugation for $15 \mathrm{~min}$, the pellet was washed once with $70 \%$ ethanol, and the air-dried pellet was dissolved in $20 \mathrm{ul}$ of $0.1 \mathrm{x}$ TE buffer $(0.001 \mathrm{M}$ Tris-HCI, 0.0001 M EDTA $[\mathrm{pH} \quad 8.0])$. For amplification of genomic DNA, PCR technique was performed by designing primers for amplifying rров (RIF- R) gene; FP: 5TACGGTCGGCGAGCTGATCC -3, RP: 5GGTTCATCGAAACGCCGTA -3. PCR reactions were performed using $1 \mathrm{mM}$ of $\mathrm{MgCl} 2,200 \mathrm{nmol}$ of each primer, $100 \mathrm{mM}$ of dNTPs, 1X PCR buffer and $10 \mathrm{ng}$ of genomic DNA with 1 unit of
Taq Hot Star DNA Polymerase Enzyme (Vivantis). The PCR program was as follows: denaturation at $94 \mathrm{C}$ for $2 \mathrm{~min}$, followed by 350 cycles at $94 \mathrm{C}$ for $30 \mathrm{~s}, 55 \mathrm{C}$ for $30 \mathrm{~s}$ and $72 \mathrm{C}$ for $30 \mathrm{~s}$, and a final extension time of $7 \mathrm{~min}$ at $72 \mathrm{C}$. Then sequences were sent to South Korea, Bioneer Corporation, for sequencing using Sanger sequencing method. SPSS statistics software was used to perform statistical operations using chisquare or non-parametric tests.

\section{RESULTS}

In this study, $55.6 \%(n=15)$ and $44.4 \%(n=12)$ of the patients were males and females, respectively. The mean age of patients was $45.59 \pm 9.04$ years. The youngest and the oldest patients were 24 and 57 years old, respectively. Among 27 positive cases, 8,11 and 8 were from Iran, Afghanistan and Turkmenistan, respectively.

In 26 samples out of $27, r p o B$ gene mutations were detected. In fact, the evaluation of gene mutations in $96.3 \%$ of the cases revealed MDR TB isolates. The most prevalent mutations belonged to the TCG (Ser) 531TTG (leu) with prevalence $51.8 \% \quad(\mathrm{n}=14), \quad$ and $\mathrm{GAC}$ (Asp)516TAC (Tyr), CAC (His) 526GAC (Asp) and CAC (His) 526TAC(Tyr) mutations with prevalence $14.8 \%(\mathrm{n}=4)$ (Table 1$)$.

On the other hand, 23, 2 and 1 patients had 1,2 and 3 mutations, respectively. However, one patient had no mutation. Ten patients had history of contact with TB patients, and 17 remaining cases did no show such history. Occupationwise, $33.3 \%(n=9)$ of the participants were housewife followed by workers $(n=5)$ and farmers $(n=3)$. As well as from each thejob training, employee job and unemployed people, two patients were included. Of boutique, bakery, confectionary and mobile sailing jobs, one patient included in the current study. In 14 cases, transmission of tuberculosis occurred at home. In 4 cases, its transmission occurred at the workplace, and one case in school. Finally, in 8 cases, the transmission route of tuberculosis was unknown. In total, the mutation in codons; 531, 526, 516, 522 and 533 were $55.5 \%(n=15), 33.3 \%(n=9), 14.8 \%(n=4)$, $3.7 \%(\mathrm{n}=1)$ and $3.7 \%(\mathrm{n}=1)$, respectively. The common sits of mutations highlighted with other colors and the color of primers was violet in Figure1.

DOI: http://dx.doi.org/10.4314/ejhs.v28i3.7 
Table 1: The pattern of $r p o B$ gene mutations in patients with MDR-TB.

\begin{tabular}{lcc}
\hline type of Mutation & Numbers of patients & Percentage (\%) \\
\hline TCG(Ser)531TTG(Leu) & 13 & 48.1 \\
GAC(Asp)516TAC(Tyr) & 1 & 3.7 \\
CAC(His)526GAC(Asp) & 2 & 7.4 \\
TCG(Ser)522CAG(Gln) & 2 & 3.7 \\
CTG(Leu)533CCG(Pro) & 1 & 3.7 \\
CAC(His)526TAC(Tyr) & 4 & 14.8 \\
TCG(Ser)531CAG(Gln) & 1 & 3.7 \\
GAC(Asp)516TAC(Tyr) & 1 & 3.7 \\
CAC(His)526GAC(Asp) & 0 & 0 \\
TCG(Ser)531TTG(Leu) & 1 & 3.7 \\
GAC(Asp)516TAC(Tyr) & 0 & 0 \\
CAC(His)526GAC(Asp) & 0 & 0 \\
GAC(Asp)516TAC(Tyr) & 1 & 3.7 \\
CAC(His)526AAC(Asp) & 0 & 0 \\
No mutation & 1 & 3.7 \\
\hline
\end{tabular}

CGTCGTCGACGCTGACCGAAGAAGACGTC GTGGCCACCATCGAATATCTGGTCCGCTTG CACGAGGGTCAGACCACGATGACCGTTCC GGGCGGCGTCGAGGTGCCGGTGGAAACCG ACGACATCGACCACTTCGGCAACCGCCGC CTGCGTACGGTCGGCGAGCTGATCCAAAA CCAGATCCGGGTCGGCATGTCGCGGATGG AGCGGGTGGTCCGGGAGCGGATGACCACC CAGGACGTGGAGGCGATCACACCGCAGAC GTTGATCAACATCCGGCCGGTGGTCGCCG CGATCAAGGAGTTCTTCGGCACCAGCCAG CTGAGCCAATTCATGGACCAGAACAACCC GCTGTCGGGGTTGACCCACAAGCGCCGAC TGTCGGCGCTGGGGCCCGGCGGTCTGTCA CGTGAGCGTGCCGGGCTGGAGGTCCGCGA CGTGCACCCGTCGCACTACGGCCGGATGT GCCCGATCGAAACCCCTGAGGGGCCCAAC ATCGGTCTGATCGGCTCGCTGTCGGTGTAC GCGCGGGTCAACCCGTTCGGGTTCATCGA AACGCCGTACC

Figure 1: The common sites of mutations have shown

\section{DISCUSSION}

Rifampin and isoniazid have the greatest anti-TB treatment potency and during the first 2 months of treatment can destroy more than $99 \%$ of tuberculosis bacilli. Therefore, the emergence of drug-resistant strains to either of these two antibiotics will bring further difficulties. Rifampin is considered as one of the most important anti-TB drugs, which by binding to beta-subunit of RNA polymerase, prevents from transcription initiation. Mutation in $r p o B$ gene encoding the beta subunit of RNA polymerase enzyme can change the spatial form of the enzyme and leads to resistance to rifampin. In recent years, several new techniques have been introduced to identify both the genotypic and phenotypic drug resistance. The advantages of genotypic methods are: shorter time-tested, no need to grow the organism in culture, the possibility of using clinical samples directly and less biological risk. However, all mechanisms are not known which lead to drug resistance (9). As mentioned in the results section, the average age of patients participating in the study was $45.59 \pm 9.04$ years. Inconsistent with our results, in a study conducted by Ramirez and et al., in 2010 (10), 154 resistant strains to rifampin were examined, the results showed the sensitivity and specificity of $91 \%$ and $98 \%$, respectively. Also, resistance rate to Rifampin and INH was $87 \%$ and $100 \%$, respectively. The sensitivity and specificity for tracing of MDR-TB strains were reported $85 \%$ and $98 \%$, respectively. In another study carried out by GoEun Choi, et al in 2010, M. tuberculosis strains were collected in three groups. The results

DOI: http://dx.doi.org/10.4314/ejhs.v28i3.7 
were as follow: All strains except one case which was resistant to rifampin, alongside all susceptible strains to rifampin were identified correctly by analyzing HRMA and $r p o B$ gene (11), that it is in line with our study. Also a study conducted by Malhotra et al., 2015; HRMA sensitivity and specificity in the detection of resistance to rifampicin were reported $90.3 \%$ and $97.4 \%$, respectively (12). Due to the geographical location of Iran and its neighboring with countries with a high prevalence of TB and because of high immigration to Iran, this study aimed to investigate the efficacy and specificity of rpoB gene sequence, mutations, and polymorphisms of this gene for the detection of isolates resistant to rifampin. In our study, the most common mutations were observed in codon 531, $526,516,533$ and 522, respectively, which was in line with a study conducted by Silva et al., 2013; in which the most common mutation was reported in rpoB gene at codon 531 and 526 (9).

In this study, the method of determining the resistance to rifampin was proportional in which the resistance level was not determined, and only resistant or susceptible modes were determined. All rроB mutations cannot cause RMP resistance in all M. tuberculosis hosts, and the occurance mutations in some parts of the gene can cause mutation. In more than $95 \%$ of cases, the mutation occurs in one or more of these three mentioned sits. Overproduction of mutated rpoB cannot cause a high level of resistance to RMP, as mutation causes interruption of antibiotic binding. Consequently, the bacterium will resistant, and it does not affect the expression or production of the product.

In this study, 23 patients with 1 mutation, 2 people with 2 mutations, 1 patient with 3 mutations, and in 1 case, no mutation was found. Similar results have been reported by global studies conducted in this field. In a study conducted by Bobadilla et al., in 2001 on rifampin-resistant strains, they were not observed any mutations in the rpoB gene(13), which it is in line with our results. Also, in a study carried out by Ohno et al., 1996; among rifampin resistantstrains of Mycobacterium tuberculosis, in one case, there was no mutation in the rpoB gene(14). In addition, a study conducted in India (Marin and et al., 2004) has found one isolate with no mutation. In our study, of 27 strains, 1 case had no mutation in the $r p o B$ gene, which is in line with the above studies, and the mentioned strain was from Iran.
Also, 1 out of rifampin-resistant M. tuberculosis strains with 3 mutations was from Iran. According to these results, it may be concluded that diversity and numbers of mutations in the codon of $r p o B$ gene in rifampin-resistant $M$. tuberculosis in Iranian patients are more than neighboring countries such as Turkmenistan and Afghanistan. The most mutations in Iranian race in $36.3 \%$ cases were observed at codon 531 and 526. Also mutation at codon 516 was observed in the $18.2 \%$ cases and $9.2 \%$ of cases had no mutation. Mutations at codon 531, 516, 526 were similar to Afghanistan race, and mutations in codon 531 and 526 were similar to the Turkmenistan race. This difference might be due to the great migrations in recent years from neighboring countries with high prevalence of TB to Iran. In recent years, the marriage of Iranian with foreigners from some countries with high burden of tuberculosis has become more common, which leads to transmission of resistant strains of M. tuberculosis to Iran. In this study, the most common mutation was observed at TCG (Ser) 531TTG (leu) in 14 cases (51.8\%).

According to our results, a high prevalence of mutations in the rpoB gene confirms that its study is suitable for detection of rifampin resistance in $M$. tuberculosis isolated from Iranian clinical samples. These results also assist us in choosing different regions of a gene to study drug resistance that is more appropriate. The use of genotypic methods for detection of rifampin-resistant strains of MBT compared to phenotypic methods is affordable and useful because the results introduce in a shorter time. On the other hand, this method can be performed directly on samples and prevents from the side pollution, so it is recommended as a fast method to start treating the patients. Rifampin-resistant strains are often associated with mutations at codon 531 of $r p o B$ gene (9), in $36.3 \%, 58.4 \%$ and $57.2 \%$ of Iranian, Afghan and Turkoman races. This is particularly important in the use of new drugs to treat tuberculosis (15). Although heterogeneity in $M$. tuberculosis may obscure the results of molecular tests used for determining the antibiotic resistance(16), however, the obtained results are valuable. In the present study, the most common mutations were reported at codon 531, and other mutations were observed at codon 526, 516, 522 and 533 , respectively. Our results are inconsistent with many global studies. In Turkmen patients, mutation at codon 516 was not observed. The mutation in this

DOI: http://dx.doi.org/10.4314/ejhs.v28i3.7 
codon was only in Iranian and Afghan patients. It can be concluded that the first step in the evaluation of antimicrobial resistance to rifampin in Turkmen patients is not needed to check codon 516, and is better at first, be checked codon 531 and 526. With detection of the only codon, 531 can detect about $63 \%$ of TB resistant strains to rifampin in the region. With detection of the codons 531 and 526 can identify approximately $72.6 \%$ of TB resistant strains to rifampin in Iranian patients.

The use of rpoB gene sequencing led to the lack of the need for growth of the organism in the culture medium, the direct use of clinical samples, and reduction of biological risks. Detection nearby $96.3 \%$ of MDR TB cases using this method lowering the cost of the treatment.

\section{ACKNOWLEDGEMENT}

This article extracted of thesis (Code 3290).

\section{REFERENCES}

1. Organization WH. Global Tuberculosis Report 2014 (World Health Organization). Geneva; 2014.

2. Bennett JE, Dolin R, Blaser MJ. Principles and practice of infectious diseases: Elsevier Health Sciences; 2014

3. Chiang CY, Centis R, Migliori GB. Drug-resistant tuberculosis: Past, present, future. Respirology. 2010;15(3):413-32.

4. Punina N, Makridakis N, Remnev M, Topunov A. Whole-genome sequencing targets drug-resistant bacterial infections. Human Genomics. 2015;9(1):1.

5. Heemskerk AD, Bang ND, Mai NT, Chau TT, Phu $\mathrm{NH}$, Loc PP, et al. Intensified Antituberculosis Therapy in Adults with Tuberculous Meningitis. New England Journal of Medicine. 2016;374(2):124-34.

6. Organization WH. Global tuberculosis report. 2016. 2016.

7. Laurenzo D, Mousa SA. Mechanisms of drug resistance in Mycobacterium tuberculosis and current status of rapid molecular diagnostic testing. Acta tropica. 2011;119(1):5-10.

8. De Freitas FA, Bernardo V, Gomgnimbou MK, Sola C, Siqueira HR, Pereira MA, et al. Multidrug resistant Mycobacterium tuberculosis: a retrospective katG and $\mathrm{rpoB}$ mutation profile analysis in isolates from a reference center in Brazil. PloS one. 2014;9(8):e104100.

9. Silva JLd, Leite GGS, Bastos GM, Lucas BC, Shinohara DK, Takinami JS, et al. Plasmid-based controls to detect rpoB mutations in Mycobacterium tuberculosis by quantitative polymerase chain reaction-high-resolution melting. Memórias do Instituto Oswaldo Cruz. 2013;108(1):106-9.

10. Li W, Matsuoka M, Kai M, Thapa P, Khadge S, Hagge DA, et al. Real-time PCR and high resolution melt analysis for rapid detection of Mycobacterium leprae drug resistance mutations and strain types. Journal of clinical microbiology. 2011: 05183-11.

11. Choi GE, Lee SM, Yi J, Hwang SH, Kim HH, Lee EY, et al. High-resolution melting curve analysis for rapid detection of rifampin and isoniazid resistance in Mycobacterium tuberculosis clinical isolates. Journal of clinical microbiology. 2010;48(11):3893-8.

12. Malhotra B, Goyal S, Bhargava S, Reddy P, Chauhan A, Tiwari J. Rapid detection of rifampicin resistance in Mycobacterium tuberculosis by highresolution melting curve analysis. The International Journal of Tuberculosis and Lung Disease. 2015;19(12):1536-41.

13. Bobadilla-del-Valle M, Ponce-de-Leon A, ArenasHuertero C, Vargas-Alarcon G, Kato-Maeda M, Small PM, et al. rpoB Gene mutations in rifampinresistant Mycobacterium tuberculosis identified by polymerase chain reaction single-stranded conformational polymorphism. Emerging infectious diseases. 2001;7(6):1010.

14. Ohno H, Koga H, Kohno S, Tashiro T, Hara K. Relationship between rifampin MICs for and rpoB mutations of Mycobacterium tuberculosis strains isolated in Japan. Antimicrobial agents and chemotherapy. 1996;40(4):1053-6.

15. Zumla A, Nahid P, Cole ST. Advances in the development of new tuberculosis drugs and treatment regimens. Nature reviews Drug discovery. 2013;12(5):388-404.

16. Zetola NM, Shin SS, Tumedi KA, Moeti K, Ncube $R$, Nicol $M$, et al. Mixed Mycobacterium tuberculosis complex infections and false-negative results for rifampin resistance by GeneXpert MTB/RIF are associated with poor clinical outcomes. Journal of clinical microbiology. 2014;52(7):2422-9.

DOI: http://dx.doi.org/10.4314/ejhs.v28i3.7 